\title{
Medical Costs of Evaluating Intellectual and Developmental Disabilities in a Unit Established in a Japanese Outpatient Office
}

\author{
Toshihiro Horiguchi ${ }^{1, *}$ and Chieko Akiyama ${ }^{2}$
}

${ }^{1}$ Department of Social Psychiatry, National Institute of Mental Health, National Center of Neurology and Psychiatry, 4-1-1 Ogawa-Higashi, Kodaira City, Tokyo 187-8553, Japan

${ }^{2}$ Akiyama Pediatric Office, 4-3-3 Kamirenjaku, Mitaka City, Tokyo 181-0012, Japan

\begin{abstract}
To determine the average medical cost incurred for early identification of intellectual and developmental disabilities in children, we retrieved the records of 264 children who had visited our consulting unit from 2005 until the end of March 2012. In the evaluation process, each child took psychological assessments and the parents were provided with solutions to enable the children to overcome their delay in school. As a whole, while the average number of visits did not differ by the diagnosis, the average medical cost differed by the diagnosis. The consultation unit was effective for gradually diminishing the medical costs incurred. Our in-clinic consultation system proved useful for early identification of intellectual and developmental disabilities in children and minimization of the medical costs incurred for the process.
\end{abstract}

Keywords: AD/HD, medical cost, PDD.

\section{INTRODUCTION}

In 2001, the Japanese Ministry of Education, Culture, Sports, Science and Technology (MEXT) published a report [1] stating that $6.3 \%$ of pupils belonging to standard schools met the criteria for special educational needs, in addition to the students in special-need settings. Although the survey results actually depended on assessment by schoolteachers using a checklist of the children's behaviors, it represented the first governmental estimation of the prevalence of children having developmental disabilities, such as attention-deficit/hyperactivity disorders (AD/HD), learning disorders (LD), and pervasive developmental disorders (PDD). Thereafter, the number of children visiting pediatricians specialized in intellectual and developmental disabilities increased throughout the nation, and this rapidly inflated demand was also named "bubble," after the name given to the remarkable rise of stock prices in Japan in the late 1980's. According to the Social Welfare Administration Duties annual report [2] published by the Ministry of Health, Labour and Welfare (MHLW), the number of consultations for children with autistic disorders increased remarkably at the public child consultation centers, exceeding 10,000 per year in 2002 , while the number of consultations fluctuated year by year.

Despite the high demand in respect of medical services for children with intellectual (mental retardation: MR) and developmental disabilities and

*Address correspondence to this author at the Department of Social Psychiatry, National Institute of Mental Health, National Center of Neurology and Psychiatry, 4-1-1 Ogawa-Higashi, Kodaira City, Tokyo 187-8553, Japan; Tel: +81-423-41-2711; Fax: +81-426-1989; E-mail: horigti@ncnp.go.jp psychological distress, consumers in Japan claim that the supply is insufficient to meet the demand. To satisfy the rising demand, in 2007, a special committee of the MHLW suggested three levels of medical settings for consultations in pediatric psychology, according to the degree of specialization [3]. According to that plan, the first level of medical attention was provided by physicians (pediatricians or psychiatrists) in general practice, the second by physicians occasionally serving in special needs, and the third by specialized physicians. Of these, the authors believed that each private pediatric office was able to contribute as a firstlevel setting to early community support for such children.

Recently, medical cost-effectiveness analyses have been published for various diseases in childhood. Studies in the domain of developmental disabilities are sparse, although a US study [4] found that cooccurrence of intellectual disability enhanced the medical expenditure for children with cerebral palsy. However, in Japan, all are allowed to choose their hospital freely. In addition, the early identification process for intellectual and developmental disabilities is not legally fixed, except for a proposed guideline by practitioners [5]. Therefore, it has not been easy to compare the medical costs incurred for identification among the disabilities.

According to an annual Survey of the Social Medical Treatment by the MHLW, the average medical cost per visit was 879 points (each point corresponding to 10 JPY) at a pediatric outpatient office in 2006. The report also revealed that it was 1,158 points at other clinical departments. Although the expenditure for the pediatric 
age group is more reasonable as compared to that for other age groups, this has not been analyzed from the viewpoint of use of medical services by children with intellectual and developmental disabilities. Assuming the above-mentioned prevalence of $6.3 \%$, we need to optimize the identification process for the near future by grasping the actual situation.

The US study referred to above retrieved Medicaid data from the public database. Nevertheless, in Japan, the annual reports of the MHLW have no specific data about the medical costs or expenditure incurred for the diagnosis of developmental disabilities. The governmental board recommended the use of the National Database (receipt information, specific medical examination and information database) after 2013. Trial analyses to explore nationwide usage of medical services in several specific diseases have just started.

In this background, the authors established a consultation unit exclusively for evaluating children with special needs or needing psychological supports in a pediatric office. To popularize our system, we assessed the unit from the viewpoint of the medical costs and expenditures.

\section{METHOD}

We initiated an in-clinic consultation unit additionally at the pediatric office managed by the second author in April 2005. The office has no beds, and is located in the western part of the Tokyo metropolitan area where the potential size of the population with medical needs is estimated to be approximately 920,000 (data of 2000).

The consultation schedule was fixed on an ondemand outpatient basis, separately from daily examination, and everyone in the community was welcome to make an appointment to visit. Parents of children who consulted pediatricians at the daily examination unit were also recommended to use the unit on another day. The collaborating psychologists worked for various problems of children caused by developmental disabilities or psychological disorders. In each session, children received routine assessments, and parents and specialists arrived at solutions (Figure 1).

For this study, the authors retrieved the medical records (paper-based chart) of 250 children who had visited the unit until the end of March 2012 (financial year), and collected data including age, gender, date, complaint, principal diagnosis, intelligence or developmental quotient (IQ or DQ), cost and paid fee for each child. Data of 14 children for whom the series of consultations started before 2005 (consultation unit was preliminarily served) were added to those of the 250 children. In total, therefore, the records of 264 children were analyzed for this study.

In Japan, specific terms for diagnoses are often used for the insurance system. For this study, the

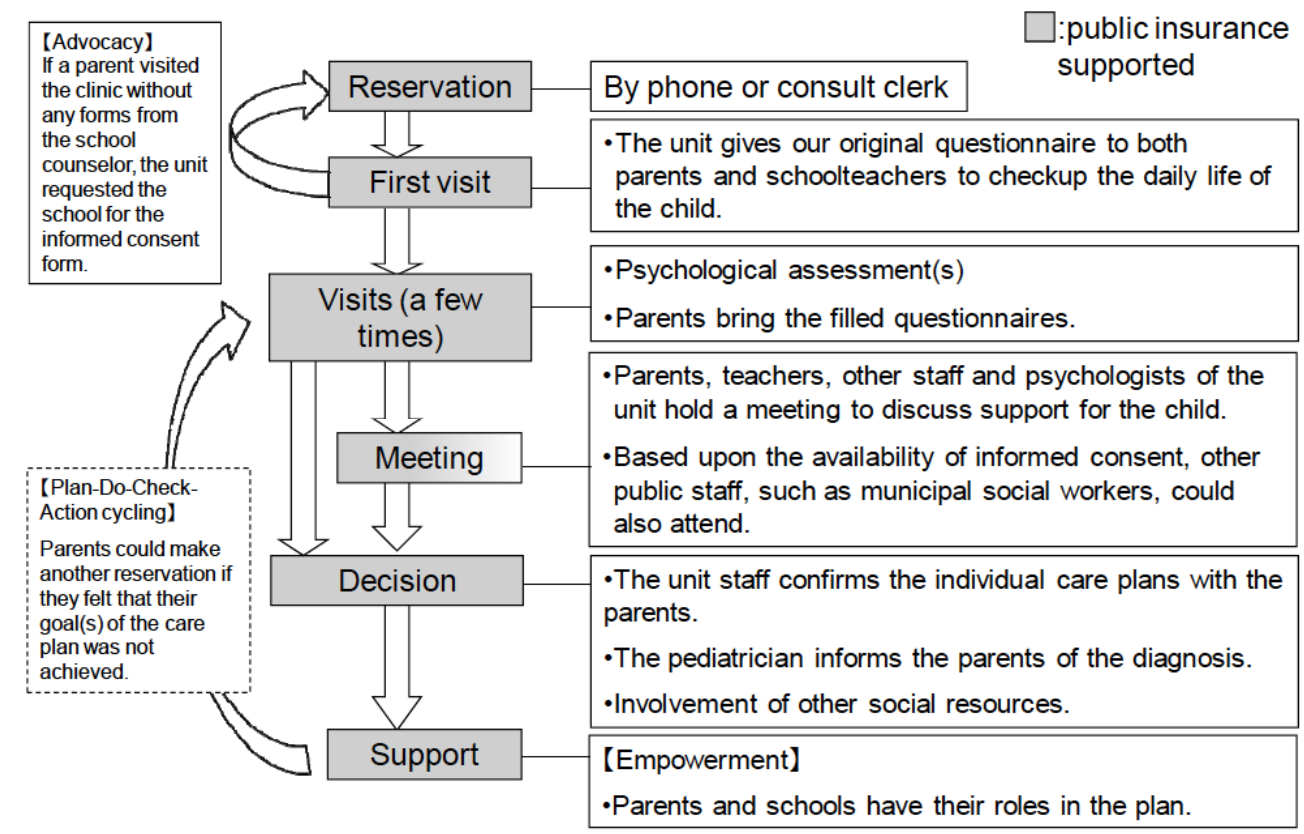

Figure 1: Flow of processes at the evaluation unit. 
diagnoses were classified by the International Classification of Diseases (ICD-10).

The duration of consultation was defined as the interval, in days, between the first visit and the latest visit, because children with intellectual and developmental disabilities require special attention even if they seek help for common diseases. Re-visits after completion of the first session were counted as unsolved complaints.

At each visit, the type of care given by the medical staff was classified according to the public classification in the MHLW report of Survey of Medical Care Activities in Public Health Insurance [6].

In Japan, medical expenditures are decided by a fee-for-service system. Points are regulated in codes representing every medical service provided by specialists. For example, the fee for pediatric counseling is 500 points and that for assessment by Wechsler's scale is 280 points, in addition to the points for basic examination. One point corresponds to 10 JPY. Medical expenses are finally decided based on the total number of points determined for each visit, and usually the insurers (an employer of parents or the city) pay $70 \%$ of the expenditure. The remaining $30 \%$ of the total expenditure is billed to the patients. However, in the later years of this study, the support for medical expenses provided by the cities increased. In the city where this investigation was carried out, care for infants (aged below 6 years) is free, and for each visit by a schoolgoing child (except from high-income homes), the amount that a family has to pay is 200 JPY. As a consequence, medical costs are measured by two parameters: points and expenditure.

Data sets were prepared anonymously at the office, and the procedure involved no violation of privacy of each child. This study was conducted with the approval of the ethical board of the institution to which the first author was affiliated (approval ID: 20-4-pre).

\section{FINDINGS}

Each year, the majority of newcomer children (105, $39.8 \%$ ) were those diagnosed as having PDD. Despite the diversity of diagnoses, distribution revealed the significance (Qui square $(\mathrm{df}=352$ ) $=566.37, \mathrm{p}<$ 0.0001). Table 1 summarizes the data of children classified by their diagnoses.

Overall, the average frequency of visits was 5.18 , and the duration of consultation was 284.95 days.

The number of visits was the highest (7.77) for cases with AD/HD. However, the duration of consultation and frequencies of visits did not differ by the diagnosis. Most of the children completed the consultation in a single session.

The average medical cost was $3,679.22$ points. Both the total points (Kruskal-Wallis $(\mathrm{df}=32) \mathrm{p}=0.03$ ) and the expenditure $(p=0.04)$ differed slightly by the

Table 1: Profiles of Children (Sorted by Principal Clinical Diagnosis ${ }^{1}$ )

\begin{tabular}{|c|c|c|c|c|c|c|c|c|}
\hline $\begin{array}{c}\text { Diagnosis (ICD-10 } \\
\text { code) }\end{array}$ & Whole & PDD (F84) & MR (F70) & AD/HD (F90) & MR,PDD & $\begin{array}{l}\text { School } \\
\text { phobia } \\
\text { (R468) }\end{array}$ & $\begin{array}{l}\text { Psychoso } \\
\text { matic (F45) }\end{array}$ & LD (F81) \\
\hline Number (persons) & 264 & 105 & 35 & 22 & 17 & 22 & 10 & 3 \\
\hline $\begin{array}{l}\text { Age at the use of the } \\
\text { unit (y.o.) }\end{array}$ & 8.12 & 7.66 & 8.21 & 8.16 & 6.92 & 12.01 & 8.96 & 8.14 \\
\hline $\begin{array}{l}\text { Proceeding times to } \\
\text { use the unit[2] (years) }\end{array}$ & 0.86 & 0.86 & 0.49 & 1.53 & 0.52 & 0.94 & 1.29 & 2.52 \\
\hline Times of visit & 5.18 & 4.30 & 2.14 & 7.77 & 3.76 & 2.55 & 23.8 & 1.33 \\
\hline $\begin{array}{l}\text { Duration of care } \\
\text { (days) [3] }\end{array}$ & 284.95 & 345.92 & 137.03 & 299.14 & 359.00 & 43.95 & 672.10 & 18.67 \\
\hline IQ, DQ [4] & 87.29 & 96.45 & 66.14 & 98.11 & 48.20 & 93.00 & 129.00 & 68.00 \\
\hline Points & $3,679.22$ & $3,154.12$ & $1,746.38$ & 3307.19 & $2,898.88$ & $1,901.86$ & $16,088.20$ & $1,534.33$ \\
\hline Expense[5] (yen) & $5,535.73$ & $5,508.48$ & $2,190.88$ & $3,629.52$ & $5,910.59$ & $4,241.82$ & $15,634.00$ & 770.00 \\
\hline
\end{tabular}

${ }^{1}$ The diagnostic term is not identical to that used for medical insurance registration. In the case of children with multiple diagnoses, the principal diagnosis was used. The table shows the diagnostic groups including $\geqq 10$ children.

${ }^{2}$ Average number of years between visit to the daily examination unit and visit to the consultation unit. This value was 0 in cases who visited the consultation unit directly.

${ }^{3}$ Average number of days between the first visit and the latest visit to the consultation unit.

${ }^{4}$ The values of quotients such as $\mathrm{IQ}, \mathrm{DQ}$ and $\mathrm{SQ}$ (social quotient) were averaged.

${ }^{5}$ Average fee billed to the parents at the office. In Mitaka city, in which this research was carried out, the municipal support for the medical expense made the expenditure zero ( $100 \%$ discounted) for preschool infants and up to 200 JPY for school-aged children. 


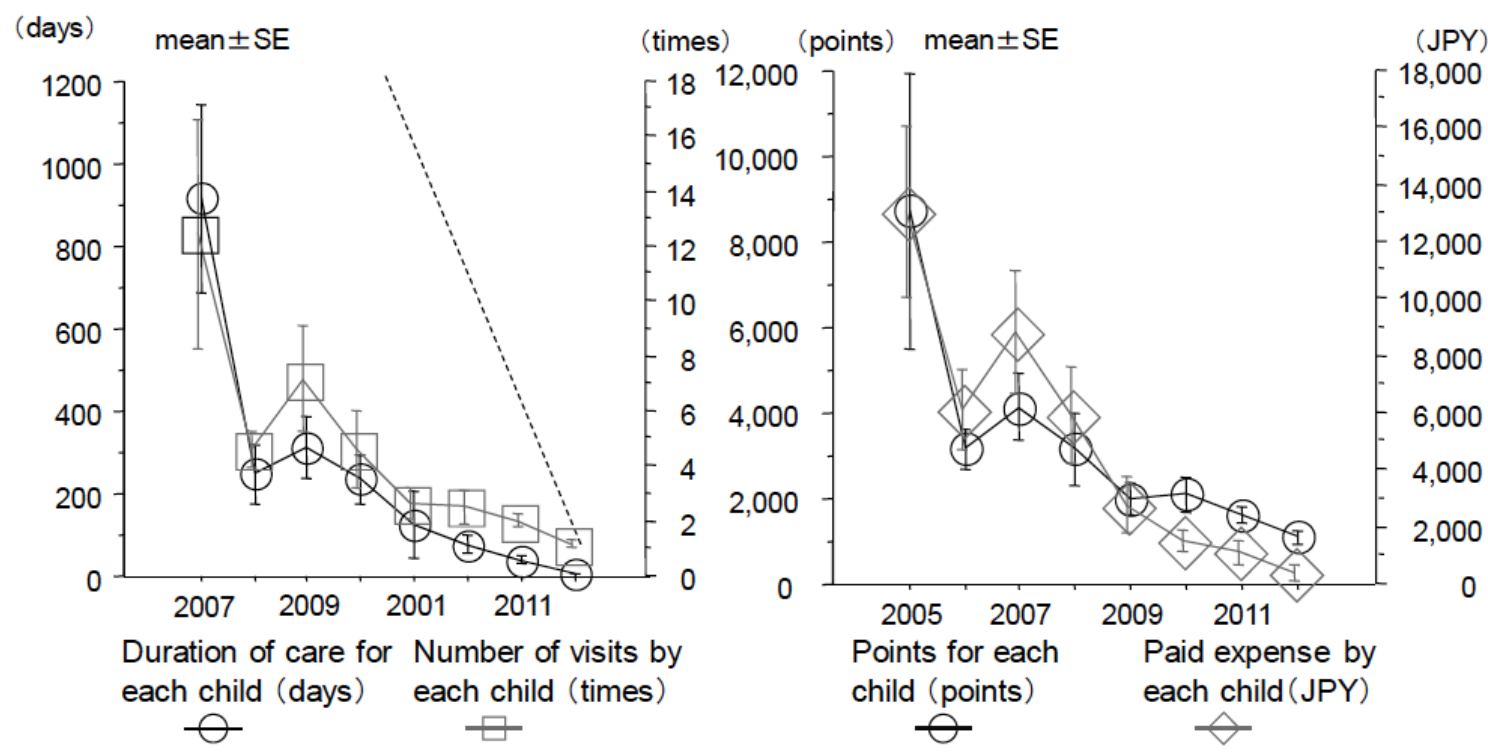

Figure 2: Duration of care and medical cost (compared by year of the first visit).

(Left)

Graphs showing the data of children who visited the consultation unit after 2005. The broken line delineates the hypothetical number of days between every new year's day and March 31, 2012. If all the children visited on new year's day and on March 312012 , the solid line would overlap the broken line. The greater the distance from the broken line, the greater the number of children who did not visit the unit for a long duration.

(Right)

Actual expenditure (pink unfilled circle) for alternative choices without medical insurance represented the fee for making a document, vaccine.

diagnosis in spite of the diversity of diagnoses. In regard to children with psychosomatic disorders, although the number of cases was few, whereas the durations were longer and the costs were higher. Children with $\mathrm{AD} / \mathrm{HD}$ started the consultation after daily examination in many cases, and the duration was shorter than in cases of PDD (and MR+PDD). The durations were shorter and the costs higher in children with school phobia.

The consultation unit managed to gradually diminish the frequencies of visits, the duration and the medical costs. Both the total points (Kruskal-Wallis, $\mathrm{df}=11, \mathrm{p}<$ $0.0001)$ and expenses $(p<0.0001)$ differed by the firstvisit year.

\section{DISCUSSION}

We analyzed the medical costs involved in identifying intellectual and developmental disabilities by collecting the records of patients at a pediatric office equipped with an in-house consultation unit.

Comparison of the medical costs incurred among the disabilities was not available due to the diverse systems in place at medical facilities in Japan. Our data enabled us to estimate the cost and expenditure involved in the diagnosis of intellectual and developmental disabilities in the community.

There were a number of consultations for children with PDDs, which is in agreement with the trend noted at the public child consultation centers mentioned in the Introduction section above. It would appear that children having PDDs without intellectual disabilities noticed as to their special needs when they experience psychological distress in standard schools. Our unit might meet the demands of families having children with such difficulties.

Due to our definition of the duration, children had long duration. For example, a child, whose initial visit was in 1998, had over 10 year duration if he/she revisited recently. The maximum duration never exceeded the value of the previous years multiplied by 365. Provided that all of the children repeated their visits without any dropouts, the duration was distributed proportionally according to the initial year of visit. The broken line descending to the right bottom in Figure 2 (left) represents this hypothesis.

However, our data did not trace the broken line precisely, since the interval between visits per child 
decreased from approximately 300 days in 2005 to 100 days in 2008. In the early years after establishment of the unit, there were fewer children and the interval between visits was longer. In the later years, the number of children was larger, while the interval between visits had become shorter.

The line in Figure 2 shows that the unit effectively shortened the duration by shrinking the interval from the first consultation to the time of provision of advice for overcoming their disabilities. In addition, the fact that the line was not straight implied that not all the children discontinued the consultation after only one session. Many visited the unit again when they encountered new difficulties. Nevertheless, the problems of most of the children could be resolved with collaboration between the unit and their school.

Some of the limitations of our study were that the data were collected from only a single setting, the sample size was small, and the satisfaction level of the consumers was not assessed. In addition, meetings held in children's schools (step 4 in the Figure 1) cannot be billed in Japan. We could therefore not calculate all the costs involved in the evaluation of developmental disabilities in children.

Nevertheless, establishment of an in-clinic consultation system possibly allows early identification and minimization of the medical costs, encourages collaboration with local social resources, and empowers both the parents and children. In conclusion, we speculate that our system is effective in providing medical support to children with intellectual and developmental disabilities.

\section{FUNDING}

Publication of this article was funded by the Health and Labour Sciences Research Grants by the MHLW (H25-Psychiatry-general-007).

\section{REFERENCE}

[1] MEXT: Future vision on Special Needs Education: final report. (http://www.mext.go.jp/b_menu/shingi/chousa/shotou/ 018/toushin/030301.htm) 2003 (in Japanese, Last accessed Oct 26, 2015).

[2] MHLW: Social Welfare Administration Duties annual report. (http://www.mhlw.go.jp/toukei/list/38-1a.html) (in Japanese, Last accessed Oct 26, 2015).

[3] MHLW: Final report of the Committee on training of the specialist in pediatric psychology. (http://www.mhlw.go.jp/ houdou/2007/03/h0330-13.html) 2006 (in Japanese, Last accessed Oct 26, 2015)

[4] Kancherla V, Amendah DD, Grosse SD, Yeargin-Allsopp M, Van Naarden Braun K.. Medical expenditures attributable to cerebral palsy and intellectual disability among Medicaidenrolled children. Res Dev Disabil 2012; 23(3): 832-40. http://dx.doi.org/10.1016/j.ridd.2011.12.001

[5] Tanaka K, Horiguchi T, Inagaki M, Kaga M. [Guidelines for medical examination of children with mental retardation in pediatric clinics which are specialized for developmental disorders--recommendation based on the current selection and usefulness of diagnostic examinations for children with mental retardation and/or pervasive developmental disorder]. No To Hattatsu 2004; 36(3): 224-9. (in Japanese)

[6] MHLW: Survey of Medical Care Activities in Public Health Insurance in 2006 (http://www.mhlw.go.jp/toukei/saikin/ hw/sinryo/tyosa06/index.html) (in Japanese, Last accessed Oct 26, 2015).

\section{DOI: http://dx.doi.org/10.6000/2292-2598.2015.03.04.7}

(c) 2015 Horiguchi and Akiyama; Licensee Lifescience Global.

This is an open access article licensed under the terms of the Creative Commons Attribution Non-Commercial License (http://creativecommons.org/licenses/by-nc/3.0/) which permits unrestricted, non-commercial use, distribution and reproduction in any medium, provided the work is properly cited. 